Article

\title{
Investigating Potential Dose-Response Relationships between Vitamin D Status and Cognitive Performance: A Cross-Sectional Analysis in Middle- to Older-Aged Adults in the Busselton Healthy Ageing Study
}

\author{
Janis D. Harse ${ }^{1, *}$, Kun Zhu ${ }^{2,3}$, Romola S. Bucks ${ }^{4}$, Michael Hunter ${ }^{1,5}$, Ee Mun Lim ${ }^{2,6}$, Brian R. Cooke ${ }^{7}$, \\ John P. Walsh ${ }^{2,3}$ and Kevin Murray ${ }^{1}$ (D)
}

check for

updates

Citation: Harse, J.D.; Zhu, K.; Bucks, R.S.; Hunter, M.; Lim, E.M.; Cooke, B.R.; Walsh, J.P.; Murray, K. Investigating Potential DoseResponse Relationships between Vitamin D Status and Cognitive Performance: A Cross-Sectional Analysis in Middle- to Older-Aged Adults in the Busselton Healthy Ageing Study. Int. J. Environ. Res. Public Health 2022, 19, 450. https:// doi.org/10.3390/ijerph19010450

Academic Editor: Paul

B. Tchounwou

Received: 9 November 2021

Accepted: 27 December 2021

Published: 31 December 2021

Publisher's Note: MDPI stays neutral with regard to jurisdictional claims in published maps and institutional affiliations.

Copyright: (c) 2021 by the authors. Licensee MDPI, Basel, Switzerland. This article is an open access article distributed under the terms and conditions of the Creative Commons Attribution (CC BY) license (https:// creativecommons.org/licenses/by/ $4.0 /)$.
1 School of Population and Global Health, University of Western Australia, Perth 6009, Australia; michael.hunter@uwa.edu.au (M.H.); kevin.murray@uwa.edu.au (K.M.)

2 Department of Endocrinology and Diabetes, Sir Charles Gairdner Hospital, Perth 6009, Australia; kun.zhu@uwa.edu.au (K.Z.); eemun.lim@health.wa.gov.au (E.M.L.); john.walsh@health.wa.gov.au (J.P.W.)

3 Discipline of Internal Medicine, Medical School, University of Western Australia, Perth 6009, Australia

4 School of Psychological Science, University of Western Australia, Perth 6009, Australia; romola.bucks@uwa.edu.au

5 Busselton Population and Medical Research Institute, Busselton 6280, Australia

6 PathWest Laboratory Medicine, Department of Clinical Biochemistry, Queen Elizabeth II Medical Centre, Perth 6009, Australia

7 PathWest Laboratory Medicine, Department of Clinical Biochemistry, Fiona Stanley Hospital, Perth 6150, Australia; Brian.Cooke@health.wa.gov.au

* Correspondence: janis.harse@research.uwa.edu.au

\begin{abstract}
Low vitamin D status has been linked to adverse cognitive outcomes in older adults. However, relationships at higher levels remain uncertain. We aimed to clarify patterns of association between vitamin D status and cognitive performance, using flexible regression methods, in 4872 middle- to older-aged adults (2678 females) from the Busselton Healthy Ageing Study. Crosssectional associations of serum levels of 25-hydroxyvitamin D (25OHD) and performance in cognitive domains were modelled using linear regression and restricted cubic splines, controlling for demographic, lifestyle, and health factors. Mean \pm SD serum $25 \mathrm{OHD}$ levels were $78 \pm 24 \mathrm{nM}$ / L for women and $85 \pm 25 \mathrm{nM} / \mathrm{L}$ for men. Increasing levels in women were associated with better global cognition (linear trend, $p=0.023$ ) and attention accuracy (continuity of attention), with improvement in the latter plateauing around levels of $80 \mathrm{nM} / \mathrm{L}$ (nonlinear trend, $p=0.035$ ). In men, increasing levels of serum 25OHD were associated with better attention accuracy (linear trend, $p=0.022$ ), but poorer semantic verbal fluency (linear trend, $p=0.025$ ) and global cognition (nonlinear trend, $p=0.015$ ). We identified patterns of association between serum 25OHD levels and cognitive performance that may reflect early dose-response relationships, particularly in women. Longitudinal analyses extending through to older ages may help to clarify the nature, strength, and temporality of these relationships.
\end{abstract}

Keywords: vitamin D; 25-hydroxyvitamin D; cognitive performance; cognitive ageing; global cognition; domain-specific cognition

\section{Introduction}

Low levels of circulating 25-hydroxyvitamin D (25OHD), the standard measure of vitamin $\mathrm{D}$, have been consistently associated with poorer health outcomes including cognitive decline in older adults [1]. The relationship with cognitive decline is most often reported in relation to global cognition [2-5] but is also noted for specific cognitive domains such as executive function [6-9] and attention and processing speed $[3,4,9,10]$. It is less evident for episodic and verbal memory $[1,6,11]$. The relationship remains unclear for higher circulating levels of $25 \mathrm{OHD}$, with better performance [2-4,6,8-10,12], plateauing 
of performance [7,13-15], and poorer performance [16-18] all having been reported. The existence of nonlinear dose-response relationships may help explain mixed results from intervention studies, as no benefit could be expected from supplementing individuals with already adequate or high baseline levels.

Biological studies suggest that vitamin $\mathrm{D}$, in its active hormonal form, has a neuroprotective role in the brain $[19,20]$. Receptors for the active, hormonal form of vitamin $\mathrm{D}$, 1,25 dihydroxy-vitamin $\mathrm{D}\left(1,25(\mathrm{OH})_{2} \mathrm{D}\right)$, have been mapped in neuronal and glial cells [21] Along with maintaining intraneuronal calcium levels, vitamin $\mathrm{D}$ regulates the production of neurotrophic factors required for nerve cell growth and the release of neurotransmitters such as acetylcholine, dopamine, and serotonin [19]. It is also known to modulate inflammatory, immune, and oxidative pathways [22], some of which are associated with neuronal ageing. In vitro, $1,25(\mathrm{OH})_{2} \mathrm{D}$ stimulates phagocytosis and clearance of the beta-amyloid material that is a hallmark of Alzheimer's disease (AD) [23]. Nonlinear dose dependencies have also been demonstrated, with physiological levels conferring protection from excitotoxic insults that low and high levels do not [24,25].

There is medical agreement that $25 \mathrm{OHD}$ levels below $50 \mathrm{nM} / \mathrm{L}$ are inadequate for bone health [26]; in addition, vitamin D deficiency has been linked to numerous other adverse health outcomes including cardiovascular disease [27,28], cancers [29], stroke [27], and all-cause mortality $[30,31]$. Consensus is still lacking as to what constitutes optimal vitamin D status, reflecting the lack of understanding of the relationship across the full range of 25OHD levels. Traditional, analytic approaches in epidemiological research of categorising vitamin $\mathrm{D}$ or treating it as a simple, continuous variable are not well-suited to characterising patterns of association across the range. Contemporary regression methods, in which segments (splines) of the range are modelled separately, offer greater precision and flexibility [32]. To date, they have been applied in a limited way to vitamin D and cognition, mostly as an adjunct to traditional methods. In two European studies, the application of restricted cubic splines (RCS) identified nonlinear patterns in relation to global cognition $[14,15]$ and attention and processing speed [15]. The relationship between low serum 25OHD levels and poorer performance was reinforced while, at higher levels, performance plateaued. However, both cohorts had relatively low serum 25OHD levels such that the capacity to explore the relationship at high levels may have been limited. Conversely, in two non-European studies where serum 25OHD levels were higher, power was likely compromised by small study sizes $(N<200)$; a positive association plateauing at around $120 \mathrm{nM} / \mathrm{L}$ was reported in relation to performance on an executive function task [8], while a negative association was reported for an episodic memory task, with the nonlinear model not improving upon the linear one [18].

Our study was based on a large community cohort of middle- to older-aged adults where serum 25OHD levels were known to be relatively high. We aimed to systematically investigate patterns of association between vitamin $\mathrm{D}$ status and performance across several cognitive domains, using linear regression and restricted cubic spline analysis. We hypothesised that patterns would be nonlinear, and their reflection of potential doseresponse relationships could have important implications for health promotion policy and supplementation practice.

\section{Materials and Methods}

\subsection{BHAS Study Cohort}

The shire of Busselton, in the southwest region of Western Australia, has been the site of regular health surveys since 1966. The longitudinal Busselton Healthy Ageing study (BHAS) commenced in 2010 with the aim of characterising the physical and cognitive ageing of 'baby-boomers' within the community [33]. All residents of the City of Busselton's Local Government Area (LGA) born between 1946 and 1964 were identified from the compulsory Western Australian electoral roll and invited to participate $(N=8223)$. A total of $82 \%$ were able to be contacted and confirmed as eligible (noninstitutionalised and still living in the region), and $76 \%$ of those contacted $(N=5107)$ agreed to take part 
in the baseline assessment. All participants completed a comprehensive health and risk factor questionnaire and attended the Health Survey Centre for physical and cognitive assessments between 2010 and 2015. Ethics approval was granted from the University of Western Australia Human Research Ethics Committee (Number RA/4/1/2203).

\subsection{Serum 25-Hydroxyvitamin D Measurement}

The vitamin D status of BHAS participants was determined from fasting blood samples collected at the time of survey centre visits. Serum 25OHD levels were assayed from these samples using the Abbott ARCHITECT platform (Abbott Laboratories, Abbott Park, IL, USA), a chemiluminescent method. The inter-assay coefficient of variation was $4.0 \%$ at $57.5 \mathrm{nmol} / \mathrm{L}$ and $2.6 \%$ at $178.3 \mathrm{nmol} / \mathrm{L}$. A subsample $(n=117)$, randomly selected within three strata of 25OHD, were also assayed using the reference method of liquid chromatography-tandem mass spectrometry (LC-MS/MS), in accordance with published methods [34]. There was excellent agreement between the two methods $(r=0.94)$. Both assays are included in national external quality assurance programs.

\subsection{Cognition}

Cognitive function was assessed using the Cognitive Drug Research (CDR) computerised assessment system (Bracket Global, Reading, UK) [35]. This set of tasks assesses immediate and delayed word recall and recognition, simple and choice reaction time, digit vigilance, spatial and numeric working memory, and delayed picture recognition. Five summary scores were derived through a previously established factor analysis [36]. Accuracy components of the choice reaction time and digit vigilance tasks contribute to the continuity of attention factor, while the time taken to complete these and the simple reaction time task contribute to the power of attention factor. Likewise, quality of episodic memory, quality of working memory, and speed of memory factors were derived from accuracy and speed components of the memory, recall, and recognition tasks. Better performance was reflected by higher scores in all factors except power of attention and speed of memory, which are based on times taken to complete tasks; thus, better performance is reflected by lower scores.

In addition, pencil-and-paper tests assessed semantic and letter verbal fluency, covering domains of language and executive function, and the Mini-Mental State Examination (MMSE) [37] was used to assess global cognitive function. The National Adult Reading Test (NART-2) [38] was also administered to provide an estimate of IQ for use as a covariate in the analysis. NART assesses the ability to pronounce words that are spelt irregularly, which is an aspect of cognition that is usually well-preserved with age and injury-related changes to the brain.

\subsection{Covariates}

All covariates were selected based on their potential to confound the analysis. While most were identified from the literature, both employment status and hours spent sitting per day were included when preliminary analysis suggested they were associated with both serum 25OHD level and cognitive performance in our study cohort. Covariate information was derived from the BHAS questionnaire or from assessments undertaken at the survey centre.

Demographic factors were age, sex, estimated IQ, and current employment status. The latter was categorised as 'retired', 'employed', or 'other' (including being unemployed, being unable to work due to illness or disability, looking after the home and family, or doing voluntary work).

Lifestyle factors were body mass index (BMI), alcohol consumption, smoking status, physical activity, hours spent sitting per day, and the use of vitamin D supplements. Both BMI, calculated as weight (kilograms) divided by height squared (metres), and hours spent sitting per day were treated as continuous variables. Smoking status was categorised as 'never smoked', 'ex-smoker', 'current smoker of fewer than 15 cigarettes per day', or 
'current smoker of 15 or more cigarettes per day'. Alcohol consumption and physical activity were categorised after preliminary analysis suggested their relationships with the cognitive outcomes were not linear. Alcohol consumption was categorised as 'nil' or within quartiles of ' 0.1 to $2.5^{\prime}$ ' ' 2.6 to $8.5^{\prime}$ ', ' 8.6 to $17.9^{\prime}$ ', and ' $18+$ ' glasses per week. Physical activity was categorised as 'low', 'medium', or 'high' in accordance with scoring guidelines for the International Physical Activity Questionnaire (IPAQ) short form [39].

Other health factors and comorbidities included self-reported general health status (poor, fair, good, very good, or excellent), which was treated as a categorical variable. The presence or history of individual comorbidities including cardiovascular disease (CVD), hypertension, diabetes, depression, and anxiety was treated as a binary variable. Depression was indicated by a self-reported history of any doctor-diagnosed and treated episode of depression or a score above the standard cut-off in the nine-item Patient Health Questionnaire (PHQ-9) [40]. Anxiety was indicated from scores above the standard cut-off on either the Generalised Anxiety Disorder (GAD-7) assessment [41] or the Depression Anxiety Stress Score (DASS-21) [42].

\subsection{Statistical Analysis}

Data were excluded for four participants who self-reported diagnoses of vascular dementia or Alzheimer's disease. Further exclusions were based on missing information for serum 25OHD level $(N=30)$, any cognitive test scores $(N=110)$, or any covariates ( $N=92$ ). Our final sample comprised 4872 participants, representing $95.4 \%$ of the baseline BHAS cohort.

The vitamin D status of individual BHAS participants was measured from blood samples collected at different times of year, and circulating 25OHD levels are known to vary markedly with season. We used previously described methods to remove this seasonal effect $[43,44]$, fitting a sinusoidal model to serum 25OHD levels with week of attendance as the predictor variable. The residual value in relation to each participant was added to the mean overall serum $25 \mathrm{OHD}$ level to obtain a predicted, de-seasonalised vitamin D status for each participant.

To facilitate comparison across domains, we also standardised cognitive $z$-scores by subtracting the mean cohort scores from individual raw cognitive scores and dividing by the cohort standard deviation. IQ was estimated from the NART score, adjusting for age, sex, and years of education, in accordance with a method developed and validated in an Australian context [45]. All analyses were stratified by sex because the relationship between vitamin $\mathrm{D}$ and cognition differs between women and men [1].

Univariate analysis was initially performed for de-seasonalised 25OHD levels, cognitive outcomes, and all covariates, with means and standard deviations calculated for continuous variables, and frequencies calculated for categorical variables. The main analysis involved regression modelling to examine patterns of association between serum 25OHD levels and cognitive $z$-scores. Firstly, unadjusted, linear models were developed for each cognitive $z$-score in which serum 25OHD was treated as a simple, continuous term. We then developed four alternative, nonlinear, unadjusted models for each cognitive $z$-score, by applying restricted cubic spline (RCS) functions to serum 25OHD with three, four, five, or six knots located at recommended percentiles [32]. Akaike information criteria (AIC) were calculated with the lowest AIC identifying the most parsimonious, nonlinear model for each cognitive $z$-score (see Table S1). The likelihood ratio test was then applied to determine whether the selected RCS models improved upon the linear ones. Once the nonlinear or linear model was selected as the 'best fit' for each cognitive score, various levels of adjustment were applied such that four models were created for each cognitive $z$-score:

Model 1: De-seasonalised 25OHD only;

Model 2: Model 1 plus age and estimated IQ; 
Model 3: Model 2 plus BMI, alcohol consumption, smoking status, physical activity category, hours spent sitting per day, employment status, and the use of vitamin D supplements;

Model 4: Model 3 plus self-reported level of general health and presence or histories of diabetes, hypertension, CVD, anxiety, and depression.

We plotted the residuals for each model to check for violations of model assumptions. Least square mean cognitive $z$-scores with $95 \%$ confidence intervals were determined across the range of serum 25OHD from the fully adjusted, 'best fit' models. In graphical presentation, the $x$-axis was restricted to values of serum $250 H D$ between $30 \mathrm{nM} / \mathrm{L}$ and $150 \mathrm{nM} / \mathrm{L}$, where $98 \%$ of values lay. To aid with interpretation, we also calculated and tabulated mean cognitive $z$-scores and 95\% confidence intervals at mid-quartile levels of serum 25OHD for all models.

Finally, a sensitivity analysis was performed, whereby participants reporting use of vitamin D supplements were excluded. In all analyses, $p$-values $<0.05$ in two-tailed tests were considered statistically significant. SAS software, version 9.4 (SAS Institute Inc., Cary, NC, USA) was used for the preparation of data and to produce descriptive statistics. All regression models were developed, and graphical and tabular output was obtained in $\mathrm{R}$ version 4.0 (R Core Team, R Foundation for Statistical Computing, Vienna, Austria).

\section{Results}

\subsection{Characteristics of the Study Cohort}

The characteristics of women and men in our study cohort are presented in Table 1. Women made up 55\% of the cohort, and mean age was similar for both sexes. Serum 25OHD levels were lower in women, and vitamin D deficiency was more prevalent, although more women reported the use of supplements. Levels of physical activity and alcohol consumption were both higher in men. A further breakdown of cohort characteristics by serum 25OHD quartiles is shown in Table S2.

Table 1. Main characteristics and raw cognitive scores for the study cohort.

\begin{tabular}{ccc}
\hline & Women $(\boldsymbol{N}=\mathbf{2 6 7 8})$ & Men $(\mathbf{N}=\mathbf{2 1 9 4})$ \\
\hline Age $($ years $)$ & $57.9 \pm 5.7$ & $58.1 \pm 5.9$ \\
De-seasonalised serum $25 \mathrm{OHD}(\mathrm{nM} / \mathrm{L})$ & $78.3 \pm 24.3$ & $84.9 \pm 24.6$ \\
Vitamin D deficient $(<50 \mathrm{nM} / \mathrm{L}), n(\%)$ & $255(9.5)$ & $83(3.8)$ \\
Estimated $\mathrm{IQ}$ & $102.6 \pm 9.6$ & $102.1 \pm 10.9$ \\
Body mass index $\left(\mathrm{kg} / \mathrm{m}^{2}\right)$ & $27.9 \pm 5.5$ & $28.5 \pm 4.1$ \\
Smoking status, $n(\%)$ & & \\
Never & $1350(50.4)$ & $931(42.4)$ \\
Ex & $1094(40.8)$ & $1014(46.2)$ \\
Current $<15$ cigarettes per day & $121(4.5)$ & $101(4.6)$ \\
Current $\geq 15$ cigarettes per day & $113(4.2)$ & $148(6.8)$ \\
Nil & & $133(6.1)$ \\
0 to 2.5 & $256(9.6)$ & $286(13.0)$ \\
2.6 to 8.5 & $843(31.5)$ & $386(17.6)$ \\
8.6 to 17.9 & $715(26.7)$ & $509(23.2)$ \\
$18+$ & $597(22.3)$ & $880(40.1)$ \\
Alcohol consumption $($ glasses per week), $n(\%)$ & $267(10.0)$ & \\
Physical activity category $($ MET minutes/week), & & $330(15.0)$ \\
$n(\%)$ & & $657(30.0)$ \\
$0-599$ & $633(23.6)$ & $1207(55.0)$ \\
$600-2999$ & $1084(40.5)$ & $4.7 \pm 2.7$ \\
3000+ & $960(35.9)$ &
\end{tabular}


Table 1. Cont.

\begin{tabular}{|c|c|c|}
\hline & Women $(N=2678)$ & $\operatorname{Men}(N=2194)$ \\
\hline \multicolumn{3}{|l|}{ Employment status, $n(\%)$} \\
\hline Employed & $1567(58.5)$ & $1603(73.1)$ \\
\hline Retired & $680(25.4)$ & $427(19.5)$ \\
\hline Other & $431(16.1)$ & $164(7.5)$ \\
\hline Use of vitamin D supplements, $n(\%)$ & $435(16.2)$ & $135(6.2)$ \\
\hline \multicolumn{3}{|l|}{ Self-reported health status, $n(\%)$} \\
\hline Poor/fair & $218(8.1)$ & $210(9.6)$ \\
\hline Good & $986(36.8)$ & $907(41.3)$ \\
\hline Very good/excellent & $1474(55.1)$ & $1077(49.1)$ \\
\hline \multicolumn{3}{|l|}{ Medical history, $n(\%)$} \\
\hline Depression & $596(22.3)$ & $335(15.4)$ \\
\hline Anxiety & $158(5.9)$ & $72(3.3)$ \\
\hline CVD & $98(3.7)$ & $178(8.1)$ \\
\hline Diabetes & $161(6.0)$ & $155(7.1)$ \\
\hline Hypertension & $1023(38.2)$ & $988(45.0)$ \\
\hline \multicolumn{3}{|l|}{ Education (level completed), $n(\%)$} \\
\hline Primary or less & $30(1.1)$ & $27(1.2)$ \\
\hline Secondary, including TAFE college & $2109(78.8)$ & $1747(79.6)$ \\
\hline Tertiary & $539(20.1)$ & $420(19.1)$ \\
\hline \multicolumn{3}{|l|}{ Raw cognitive scores } \\
\hline Continuity of attention factor & $90.6 \pm 4.2$ & $90.4 \pm 3.8$ \\
\hline Power of attention factor & $1244.1 \pm 138.4$ & $1235.2 \pm 139.4$ \\
\hline Quality of working memory factor & $187.7 \pm 16.5$ & $187.8 \pm 15.2$ \\
\hline Quality of episodic memory factor & $190.1 \pm 46.2$ & $172.6 \pm 44.7$ \\
\hline Speed of memory factor & $4319.6 \pm 859.1$ & $4359.9 \pm 885.3$ \\
\hline Semantic verbal fluency & $19.0 \pm 4.8$ & $18.1 \pm 4.6$ \\
\hline Letter verbal fluency & $40.1 \pm 11.1$ & $35.6 \pm 10.8$ \\
\hline MMSE & $28.6 \pm 1.6$ & $28.4 \pm 1.6$ \\
\hline
\end{tabular}

Values shown are means \pm SD unless otherwise indicated; percentages (\%) may not always add up to 100 due to rounding. TAFE, Technical and Further Education; MET, metabolic equivalent (a measure of physical activity related to metabolic rate); CVD, cardiovascular disease, MMSE, Mini-Mental State Examination.

\subsection{Patterns of Association between Serum 25OHD Level and Cognitive Performance}

Results of the regression modelling are presented in Figures 1 and 2, as well as Tables 2 and 3. The plotting of residuals did not suggest any major violations of model assumptions. Nonlinear and linear patterns of association were identified between serum 25OHD level and cognitive performance in several, but not all, domains. Patterns varied by domain and between BHAS women and men.

\subsubsection{Attention}

A positive and nonlinear pattern of association was identified between serum 25OHD level and continuity (accuracy) of attention in women, which persisted with adjustment for all covariates (model 4 , nonlinear $p=0.035$ ). Improvements in performance were associated with increasing serum 25OHD up to levels of approximately $80 \mathrm{nM} / \mathrm{L}$, above which the relationship plateaued (Figure 1). In men, a linear pattern was identified (model 4, linear $p=0.022$ ), suggesting that improvements in accuracy of attention were associated with increasing serum 25OHD levels throughout the range (Figure 2). From the lowest to highest serum 25OHD quartiles, mean continuity of attention z-scores increased by approximately $0.13 \mathrm{SD}$ in women and 0.09 in men (Tables 2 and 3). This equated to less than one point in the raw continuity of attention factor scores for both sexes. No significant associations were observed between serum 25OHD levels and the power (speed) of attention, in either sex. 

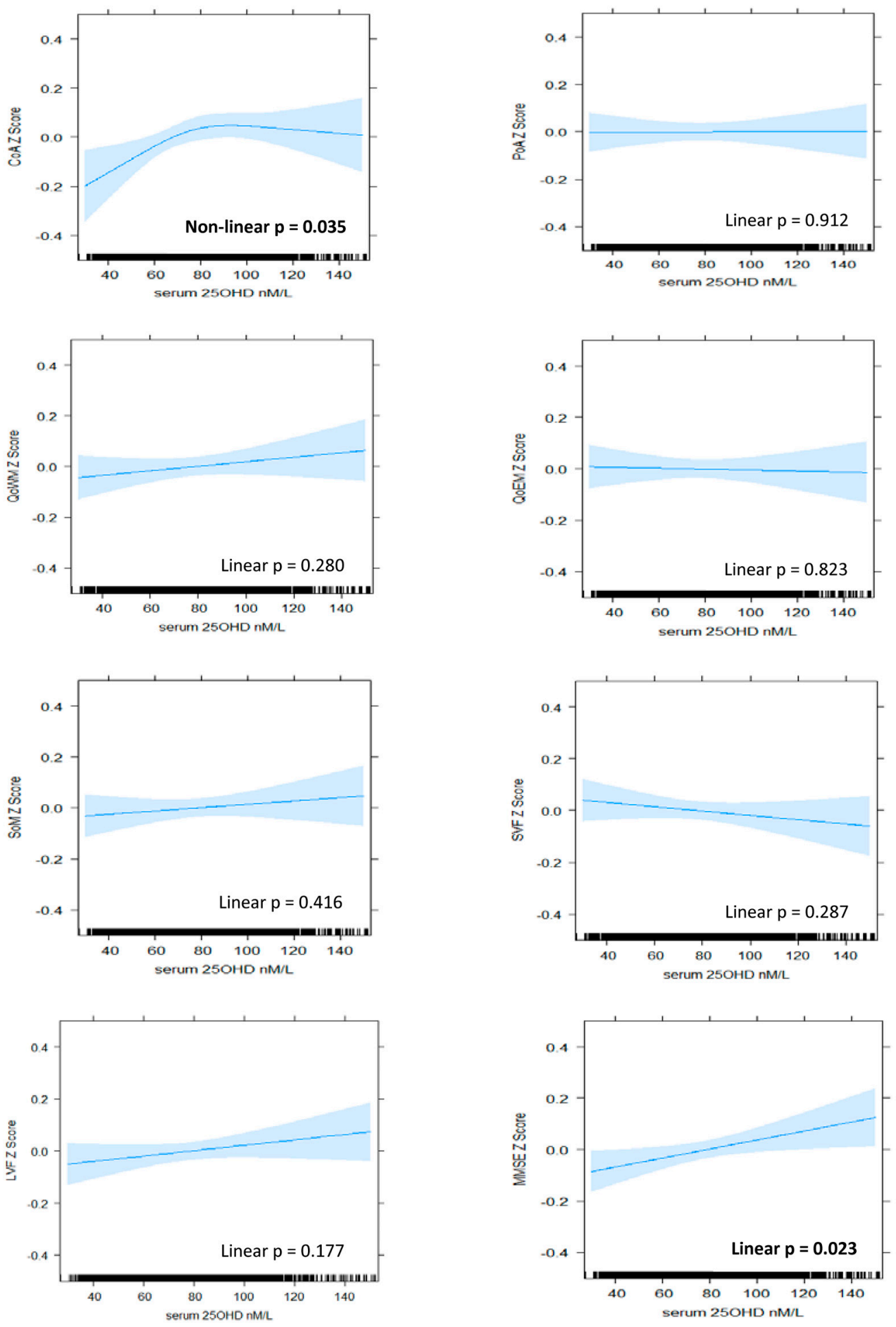

Figure 1. The association between serum 25OHD level (nM/L) and cognitive $z$-scores in BHAS women. Plots derived from 'best fit' of nonlinear (RCS) versus linear and fully adjusted models (Model 4). Model 4 includes de-seasonalised serum 25OHD level, age, estimated IQ, BMI, alcohol consumption, smoking status, physical activity, sitting hours per day, employment status, self-reported use of vitamin D supplements, self-reported health status, individual histories of hypertension, cardiovascular disease, diabetes, depression, and anxiety. CoA, continuity of attention; PoA, power of attention; QoWM, quality of working memory; QoEM, quality of episodic memory; SoM, speed of memory; SVF, semantic verbal fluency; LVF, letter verbal fluency; MMSE, Mini-Mental State Examination. 

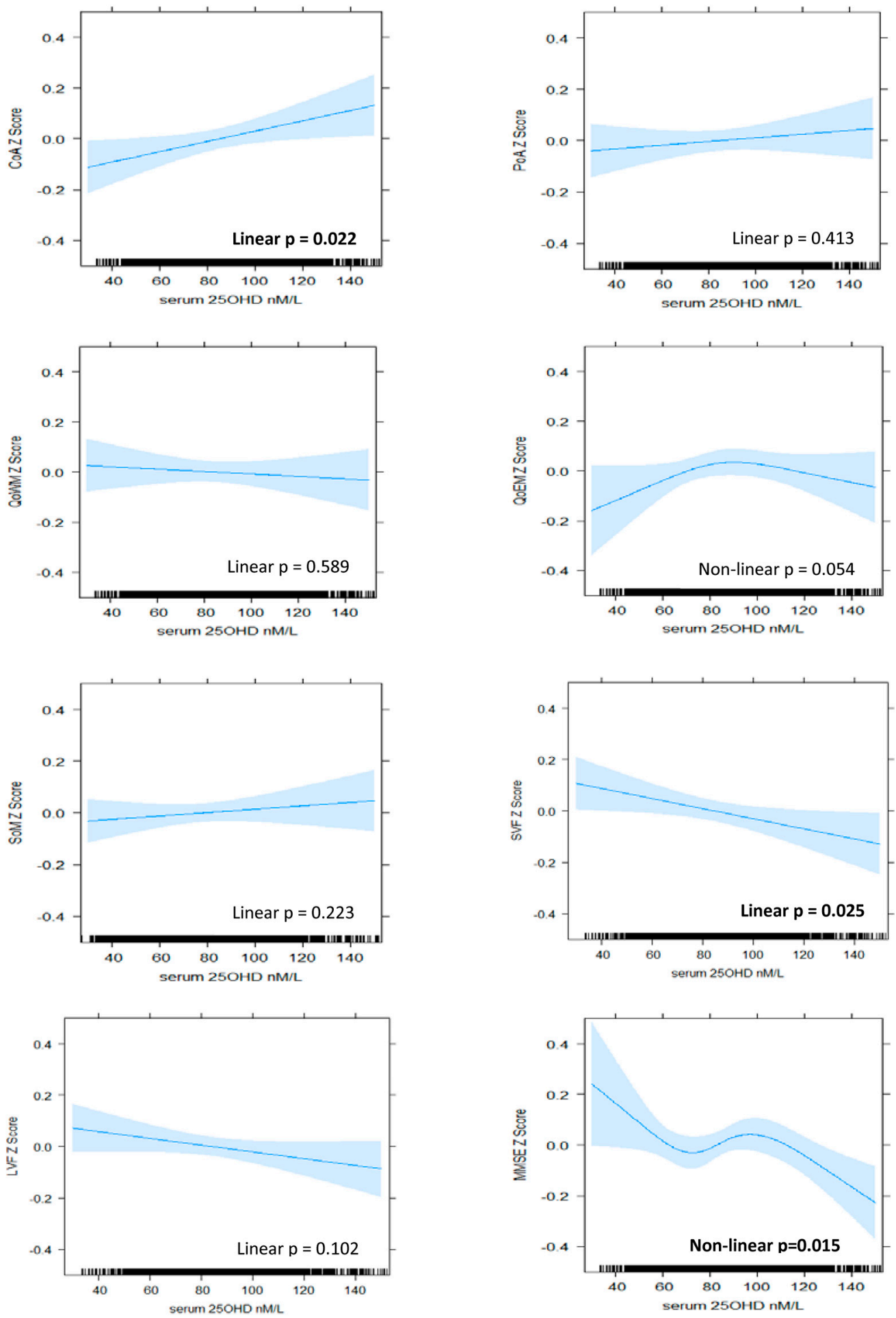

Figure 2. The association between serum 25OHD level (nM/L) and cognitive $z$-scores in BHAS men. Plots derived from 'best fit' of nonlinear (RCS) versus linear and fully adjusted models (Model 4). Model 4 includes de-seasonalised serum 25OHD level, age, estimated IQ, BMI, alcohol consumption, smoking status, physical activity, sitting hours per day, employment status, self-reported use of vitamin D supplements, self-reported health status, individual histories of hypertension, cardiovascular disease, diabetes, depression, and anxiety. CoA, continuity of attention; PoA, power of attention; QoWM, quality of working memory; QoEM, quality of episodic memory; SoM, speed of memory; SVF, semantic verbal fluency; LVF, letter verbal fluency; MMSE, Mini-Mental State Examination. 
Table 2. Cognitive $z$-scores (least square means and 95\% confidence limits) at mid-quartile levels of serum 25OHD in BHAS women derived from 'best fit' models.

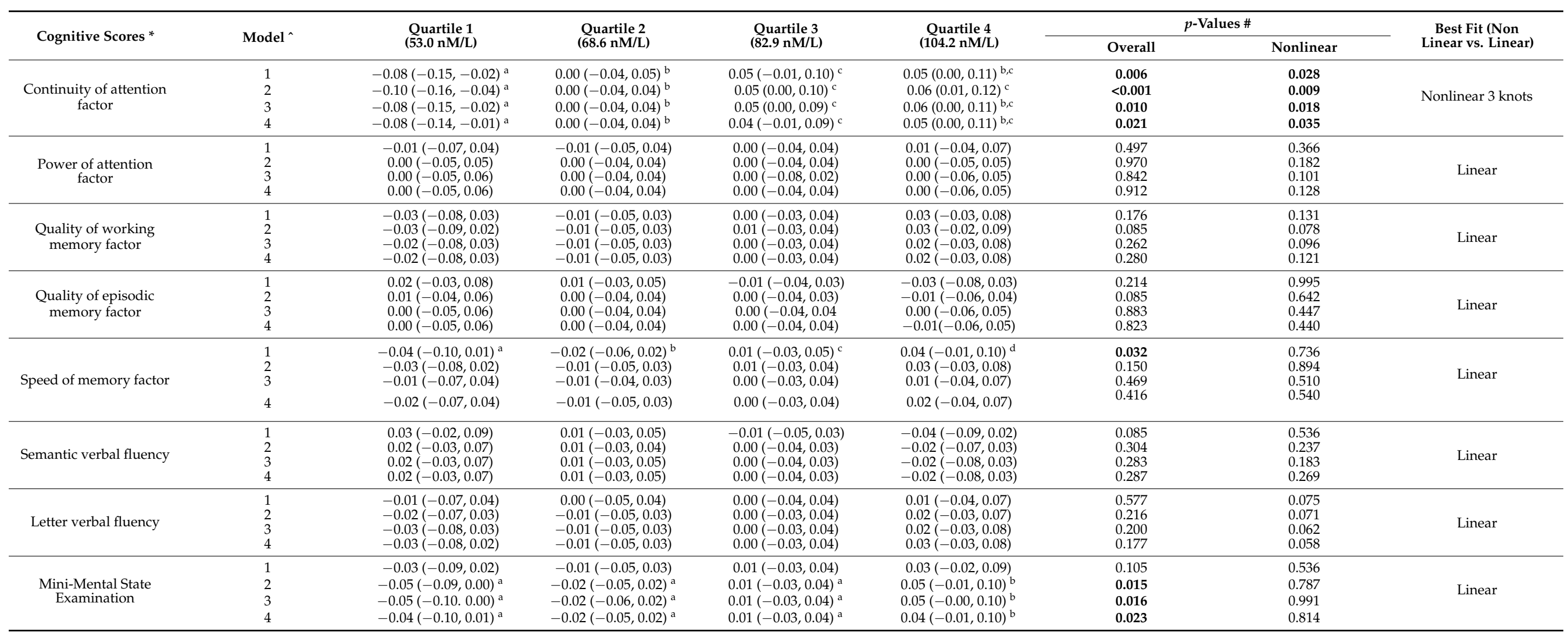

* Higher cognitive scores indicate better performance except in the case of power of attention and speed of memory, where higher scores indicate slower speed and worse performance. ^ Model 1: De-seasonalised serum 25OHD only; Model 2: Model 1 plus age and estimated IQ; Model 3: Model 2 plus BMI, alcohol consumption, smoking status, physical activity (low, medium, high), sitting hours per day, employment status (employed, retired or other), and use of vitamin D supplements; Model 4: Model 3 plus self-reported health status and history (yes vs. no) of hypertension, cardiovascular disease, diabetes, depression, and anxiety. \# $p$-Value overall: overall $p$-value for serum $25 \mathrm{OHD}$ where nonlinear model was selected, or the $p$-value for the linear term where linear model was selected; $p$-value nonlinear: $p$-value from likelihood ratio test of whether nonlinear model improves on the simple, linear model. $p$-Values $\leq 0.05$ are highlighted in bold. ${ }^{\mathrm{a}-\mathrm{d}}$ In rows with superscripts $(\mathrm{a}, \mathrm{b}, \mathrm{c}, \mathrm{d})$, mean values without a common letter indicate that means differ, $p<0.05$. 
Table 3. Cognitive $z$-scores (least square means and 95\% confidence limits) at mid-quartile levels of serum 25OHD in BHAS men derived from 'best fit' models.

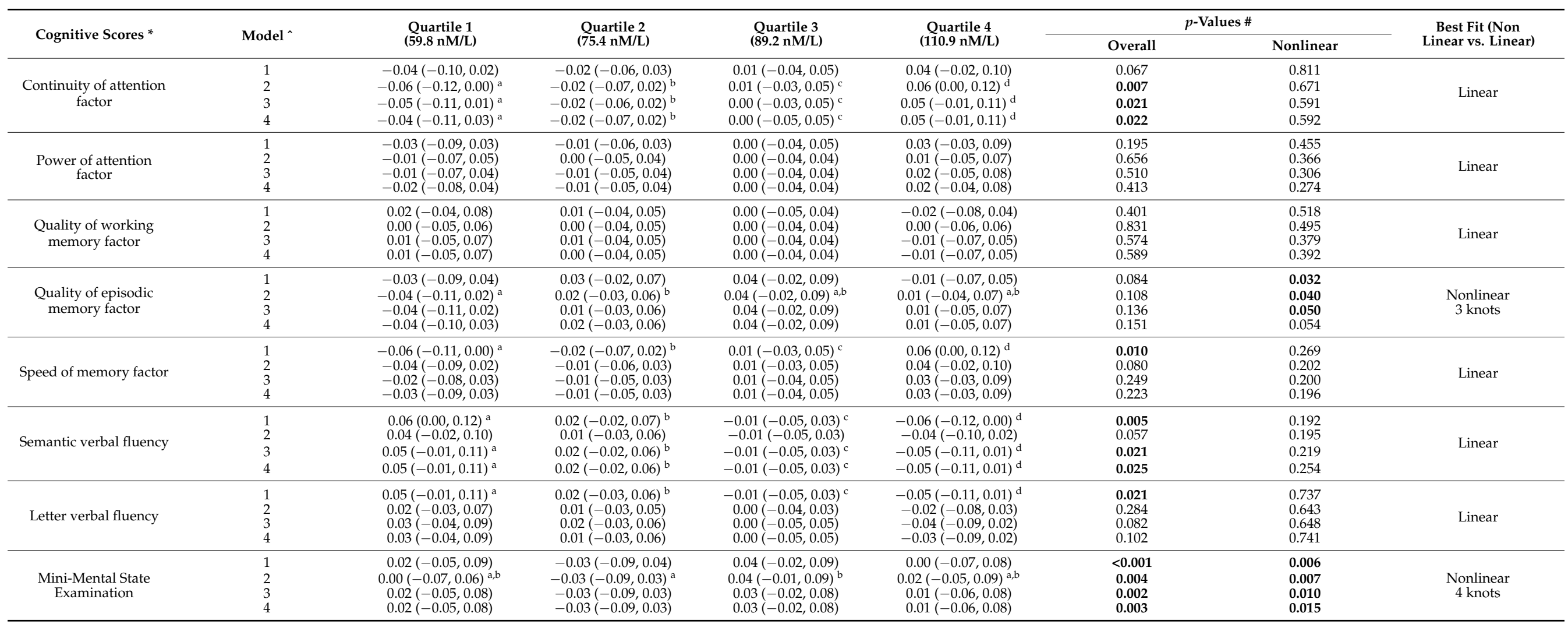

* Higher cognitive scores indicate better performance except in the case of power of attention and speed of memory, where higher scores indicate slower speed and worse performance. 'Model 1: De-seasonalised serum 25OHD only, Model 2: Model 1 plus age and estimated IQ, Model 3: Model 2 plus BMI, alcohol consumption, smoking status, physical activity (low, medium, high), sitting hours per day, employment status (employed, retired or other), and use of vitamin D supplements, Model 4: Model 3 plus self-reported health status and history (yes vs. no) of hypertension, cardiovascular disease, diabetes, depression, and anxiety. \# $p$-Value overall: overall $p$-value for serum 25OHD where nonlinear model was selected, or the $p$-value for the linear term where linear model was selected; $p$-value nonlinear: $p$-value from likelihood ratio test of whether nonlinear model improves on the simple, linear model. $p$-Values $\leq 0.05$ are highlighted in bold. ${ }^{\mathrm{a}-\mathrm{d}}$ In rows with superscripts $(\mathrm{a}, \mathrm{b}, \mathrm{c}, \mathrm{d})$, mean values without a common letter indicate that means differ, $p<0.05$. 


\subsubsection{Memory}

A positive and nonlinear pattern was identified between serum 250HD level and quality of episodic memory in men in the unadjusted model (nonlinear $p=0.032$ ). However, this was no longer statistically significant after adjustment for all covariates (model 4, nonlinear $p=0.054$ ).

For both sexes, linear patterns were observed between serum 25OHD level and speed of memory retrieval in unadjusted analyses (women, linear $p=0.032$; men, linear $p=0.010$ ), but these were also no longer significant after adjustment for age and estimated IQ (women, linear $p=0.150$; men, linear $p=0.080$ ). No association was identified between serum $25 \mathrm{OHD}$ level and quality of episodic memory in women or quality of working memory in either sex.

\subsubsection{Verbal Fluency}

A negative, linear relationship was identified between serum 25OHD level and semantic fluency in men (model 4 , linear $p=0.025$ ), suggesting that poorer performance was associated with increasing levels of serum 25OHD (Figure 2). From the lowest to highest quartiles of serum 25OHD, mean semantic fluency decreased by 0.10 SD (Table 3), equating to less than one word. No significant patterns of association were identified for semantic fluency in women nor for letter fluency in either sex.

\subsubsection{Global Cognition}

A linear pattern of positive association was identified between serum 25OHD levels and MMSE score in women (model 4 , linear $p=0.023$ ). An improvement in mean MMSE $z$-score of $0.08 \mathrm{SD}$ was associated with moving from the lowest to highest quartile (Table 3 ), which equated to less than 1 point on the raw MMSE score scale. In men, a nonlinear and overall negative pattern was identified (model 4 , nonlinear $p=0.015$ ). Better performance was associated with low levels of serum 25OHD, and poorer performance was associated with high levels, although, at mid-range levels (from $70 \mathrm{nM} / \mathrm{L}$ to $100 \mathrm{nM} / \mathrm{L}$ ), the pattern reversed such that increasing serum 25OHD level was associated with improving performance (Figure 2).

\subsection{Sensitivity Analysis}

The removal of those reporting the use of vitamin D supplements from the analyses mildly enhanced the association between serum 25OHD level and continuity (accuracy) of attention in women (model 4 , nonlinear $p=0.019$ ) and both semantic fluency (model 4, linear $p=0.009$ ) and quality of episodic memory in men (model 4 , nonlinear $p=0.031$ ). Conversely, associations with MMSE score were attenuated, more so in women (model 4, linear $p=0.092$ ) than in men (model 4 , nonlinear $p=0.016$ ), (see Supplementary Tables S3 and S4).

\section{Discussion}

Our investigation of the relationship between vitamin $\mathrm{D}$ and cognitive performance in middle- to older-aged adults highlighted several nonlinear and linear patterns of association, although effect sizes were small. Associations were more positive in women, compared with men. This is consistent with the literature which suggests that the relationship between vitamin $\mathrm{D}$ and cognitive performance is stronger for women [1]. There are physiological interactions between the effects of vitamin D and oestrogen [22], such that the postmenopausal depletion of oestrogen may render women more vulnerable to the effects of vitamin D deficiency [46].

Accuracy of attention was the only domain that was positively associated with serum $25 \mathrm{OHD}$ levels in both sexes. Lower levels, below approximately $80 \mathrm{nM} / \mathrm{L}$ in women and $90 \mathrm{nM} / \mathrm{L}$ in men, were associated with poorer accuracy of attention. Patterns diverged at higher levels, with a plateauing effect observed for women and continued improvement observed for men. In the literature, a relationship between low vitamin D status and poorer performance in older adults has been reported with respect to attention and other specific 
domains, although thresholds have varied [1]. Likewise, a divergence of patterns at higher levels is common in the literature, although not necessarily based on sex. When the same continuity of attention factor was examined in a cohort of older adults (85 years plus), moderate levels of serum 25OHD were associated with better performance than lower levels, but not higher levels [13]. A plateauing pattern was also modelled for performance in a coding task that assessed attention and processing speed [15]. In contrast, mean performance was reported to improve throughout the range of serum 25OHD levels for both the digit symbol substitution test $[3,10]$ and the digit span task [6], two other measures of attention and processing speed that also tap working memory.

We observed a significant association between vitamin $\mathrm{D}$ and global cognition (measured with the MMSE) for both sexes, but the patterns were very different. The positive and linear pattern identified for vitamin D status and global cognition in women has many precedents in the literature. Improved performance throughout the range was reported in mixed-sex studies using the MMSE [6,47], as well as other measures of global cognition such as the abbreviated mental test [5] and composite scores [3,4,48]. While nonlinear patterns have also been previously reported in relation to global cognition $[14,15]$, they do not resemble the complex pattern identified in our study for men. This pattern is difficult to interpret and lacks biological plausibility as an overall dose-response pattern. While the positive association through mid-range levels of serum 25OHD and the association between higher levels and poorer performance are consistent with some previous findings [16,17], the association between lower vitamin $\mathrm{D}$ levels and better performance runs contrary to most of the literature. Nevertheless, there is some consistency with the negative association we also observed for semantic fluency in men. Furthermore, a negative and linear pattern of association was reported between serum 25OHD level and verbal learning and memory in another Western Australian study [18]. This latter cohort was slightly older (mean 66 years), and 25OHD levels were higher (mean $=85 \mathrm{nM} / \mathrm{L}$ ) than in our cohort. A form of reverse causation was posited, whereby late middle-aged and healthy individuals were likely to be engaged in predominantly indoors, white collar work and have high cognitive function but low vitamin D status [18]. Three-quarters of the men in our cohort were still employed, and, while we adjusted for employment status and related factors such as sitting hours per day and estimated IQ, we were unable to adjust for occupation type. In addition, the variability in the lower part of the serum $25 \mathrm{OHD}$ range, where data are scarce, suggests further caution in the interpretation of this finding.

Our study had several strengths including its size and the use of RCS to systematically investigate the patterns of association between vitamin D status and cognitive performance across the range of serum $25 \mathrm{OHD}$ levels. Relatively few significant associations were identified, and effect sizes were small, suggesting the relationship between vitamin D and cognitive performance may not be strong. However, this may reflect both the relatively young age and high vitamin D status of our study cohort. The strongest associations have been previously reported where adults are over 65 years of age $[1,49,50]$. Similarly, findings are stronger in studies where vitamin D deficiency is more prevalent [6,51]. While middle age may be a critical time for maintaining adequate vitamin $\mathrm{D}$ status to protect the nervous system, the effects of depletion may not manifest until it becomes chronic in later life and there is vulnerability to other insults [52].

The pattern identified for accuracy of attention in women has particular biological plausibility with respect to the neuroprotective role suggested for vitamin $\mathrm{D}$. The distinct decline in performance associated with lower serum 25OHD levels may represent an early, subclinical sign of vitamin D-related cognitive impairment in these middle- to older-aged women. In view of the normally long, preclinical phase associated with cognitive decline, identification of this marker may provide important opportunities for early intervention. Unlike many other potential risk factors, vitamin D deficiency is easily addressed through taking supplements.

The relatively high vitamin D status of our cohort facilitated an investigation of the relationship at higher levels. Yet, as with previous studies, our findings were mixed. In 
women, levels of at least 70-80 nM/L were associated with optimal cognitive performance, and higher levels were not associated with poorer performance in any domain. Our findings were less clear for men, with increasing vitamin D status associated with both positive and negative outcomes across different cognitive domains. Therefore, we cannot make any inference regarding vitamin D status and the cognitive health of middle- to older-aged men. While other factors may be at play, the negative relationships also suggest some caution with respect to vitamin $\mathrm{D}$ supplementation of men at this age.

Due to the cross-sectional nature of this study, we cannot be sure that the identified patterns of association reflect true dose-response relationships. While we excluded known cases of dementia, reverse causation could be responsible for the positive relationships observed between vitamin $\mathrm{D}$ and cognition. Older adults with poorer cognition may get less sunlight and have a poorer diet in comparison to those with better cognition. Additionally, while we adjusted for numerous factors, some residual confounding also appears likely. We did not adjust for occupation type and were also unable to distinguish between occupational and recreational physical activity, although the latter has been more positively associated with cognitive performance in older adults [53]. Some potential confounders such as smoking status and levels of alcohol consumption may have been underestimated as they relied on self-report.

While large randomised controlled trials may be required to address causation, they will need to be of long duration and should address the potential for nonlinear relationships. Further longitudinal studies should assess serum 25OHD levels and cognitive performance at multiple timepoints from middle through to older age to better understand the temporality of the relationship. The BHAS is a longitudinal study that should provide such opportunity in the future.

\section{Conclusions}

Our study identified positive patterns of associations between vitamin D status and attention accuracy in middle- to older-aged women and men, as well as global cognition in women. While causation was not established, these patterns do have some plausibility as causal, dose-response relationships. In particular, the sharp decline in attention accuracy associated with below average vitamin D status in women appears consistent with the neuroprotective role proposed in biological studies. While no inference can be made with respect to an optimal vitamin D status in middle- to older-aged men, a level of at least 70 to $80 \mathrm{nM} / \mathrm{L}$ was associated with better cognitive health in women at this age.

Supplementary Materials: The following are available online at https://www.mdpi.com/article/10 .3390 /ijerph19010450/s1: Table S1. Akaike information criteria (AIC) comparing regression models for cognitive $z$-scores that include restricted cubic splines (RCS) with 3-6 knots for serum 25OHD level; Table S2. Characteristics of Busselton Healthy Ageing Study (BHAS) women and men across sexspecific serum 25OHD quartiles; Table S3. Cognitive z-scores (least square means and 95\% CLs) at midquartile levels of de-seasonalised serum 25OHD in BHAS women not taking vitamin D supplements, derived from 'best-fit' of linear and nonlinear (RCS) and fully adjusted models (Model 4); Table S4. Cognitive $z$-scores (least square means and 95\% CLs) at mid-quartile levels of de-seasonalised serum $25 \mathrm{OHD}$ in BHAS men not taking vitamin D supplements, derived from 'best fit' of linear and nonlinear (RCS) and fully adjusted models (Model 4).

Author Contributions: Conceptualisation, J.D.H., K.Z., R.S.B., M.H., J.P.W. and K.M.; formal analysis, J.D.H.; investigation, M.H.; methodology, J.D.H., K.Z., R.S.B., M.H., E.M.L., B.R.C., J.P.W. and K.M.; supervision, K.Z., R.S.B., M.H. and K.M.; validation, E.M.L. and B.R.C.; writing-original draft, J.D.H.; writing—review and editing, K.Z., R.S.B., M.H., E.M.L., B.R.C., J.P.W. and K.M. All authors have read and agreed to the published version of the manuscript.

Funding: The lead author (J.D.H.) is supported by an Australian Government PhD scholarship and stipend.

Institutional Review Board Statement: The study was conducted according to the guidelines of the Declaration of Helsinki and approved for this study as part of a PhD project by the UWA Human 
Research Ethics Committee (RA/4/20/5356). Ethics approval for the BHAS was granted from the University of Western Australia's (UWA) Human Research Ethics Committee (RA/4/1/2203).

Informed Consent Statement: Informed consent was obtained from all subjects involved in the study.

Data Availability Statement: Application for access to the deidentified, individual BHAS participant data underlying the results of this study can be made to the BPMRI. Applications would be reviewed by the BPMRI Scientific Committee.

Acknowledgments: The authors wish to acknowledge and thank the BPMRI for access to BHAS data, Mark Divitini and Kashif Mukhtar for their curation of BHAS data, the Busselton Health Study Centre staff, and volunteers for the survey operations, the people of Busselton for their participation in the BHAS, and the late Professor Keith Wesnes for management of the Cognitive Drug Research (CDR) computerised assessment system. The Busselton Healthy Ageing Study (BHAS) is supported by grants from the Government of Western Australia (Department of Jobs, Tourism, Science, and Innovation), the City of Busselton, and private donations to the Busselton Population Medical Research Institute (BPMRI). General infrastructure support for the BHAS is provided by the Western Australian Country Health Service. Vitamin D assay reagents and some consumables were provided for the BHAS by Abbott Australasia Pty Ltd and BD Biosciences.

Conflicts of Interest: The authors declare no conflict of interest. The funders had no role in the design of the study; in the collection, analyses, or interpretation of data; in the writing of the manuscript, or in the decision to publish the results.

\section{References}

1. Goodwill, A.M.; Szoeke, C. A Systematic Review and Meta-Analysis of The Effect of Low Vitamin D on Cognition. J. Am. Geriatr. Soc. 2017, 65, 2161-2168. [CrossRef] [PubMed]

2. Bartali, B.; Devore, E.; Grodstein, F.; Kang, J.H. Plasma vitamin D levels and cognitive function in aging women: The nurses' health study. J. Nutr. Health Aging. 2014, 18, 400-406. [CrossRef]

3. Wilson, V.K.; Houston, D.K.; Kilpatrick, L.; Lovato, J.; Yaffe, K.; Cauley, J.A.; Harris, T.B.; Simonsick, E.M.; Ayonayon, H.N.; Kritchevsky, S.B.; et al. Relationship between 25-hydroxyvitamin D and cognitive function in older adults: The Health, Aging and Body Composition Study. J. Am. Geriatr. Soc. 2014, 62, 636-641. [CrossRef] [PubMed]

4. Handing, E.P. Mediation and Moderation Analysis of Nutrition, Inflammatory Biomarkers, and Cognition in Older Adults. Ph.D. Thesis, University of South Florida, Tampa, FL, USA, January 2015.

5. Annweiler, C.; Milea, D.; Whitson, H.E.; Cheng, C.Y.; Wong, T.Y.; Ikram, M.K.; Lamoureux, E.L.; Sabanayagam, C. Vitamin D insufficiency and cognitive impairment in Asians: A multi-ethnic population-based study and meta-analysis. J. Intern. Med. 2016, 280, 300-311. [CrossRef]

6. Buell, J.S.; Scott, T.M.; Dawson-Hughes, B.; Dallal, G.E.; Rosenberg, I.H.; Folstein, M.F.; Tucker, K.L. Vitamin D is associated with cognitive function in elders receiving home health services. J. Gerontol. Ser. A Biol. Sci. Med. Sci. 2009, 64, 888-895. [CrossRef] [PubMed]

7. Annweiler, C.; Maby, E.; Meyerber, M.; Beauchet, O. Hypovitaminosis D and executive dysfunction in older adults with memory complaint: A memory clinic-based study. Dement. Geriatr. Cogn. Disord. 2014, 37, 286-293. [CrossRef]

8. Pettersen, J.A. Vitamin D and executive functioning: Are higher levels better? J. Clin. Exp. Neuroipsychol. 2016, 38, 467-477. [CrossRef] [PubMed]

9. Karakis, I.; Pase, M.P.; Beiser, A.; Booth, S.L.; Jacques, P.F.; Rogers, G.; DeCarli, C.; Vasan, R.S.; Wang, T.J.; Himali, J.J.; et al. Association of serum Vitamin D with the risk of incident dementia and subclinical indices of brain aging: The Framingham Heart Study. J. Alzheimer's Dis. 2016, 51, 451-461. [CrossRef]

10. Lee, D.M.; Tajar, A.; Ulubaev, A.; Pendleton, N.; O'Neill, T.W.; O'Connor, D.B.; Bartfai, G.; Boonen, S.; Bouillon, R.; Casanueva, F.F.; et al. Association between 25-hydroxyvitamin D levels and cognitive performance in middle-aged and older European men. J. Neurol. Neurosurg. 2009, 80, 722-729. [CrossRef] [PubMed]

11. Kueider, A.M.; Tanaka, T.; An, Y.; Kitner-Triolo, M.H.; Palchamy, E.; Ferrucci, L.; Thambisetti, M. State- and trait-dependent associations of vitamin-D with brain function during aging. Neurobiol. Aging. 2016, 39, 38-45. [CrossRef]

12. Brouwer-Brolsma, E.M.; van de Rest, O.; Tieland, M.; van der Zwaluw, N.L.; Steegenga, W.T.; Adam, J.J.; van Loon, L.J.C.; Feskens, E.J.M.; de Groot, L.C.P.G.M. Serum 25-hydroxyvitamin D is associated with cognitive executive function in Dutch prefrail and frail elderly: A cross-sectional study exploring the associations of 25-hydroxyvitamin D with glucose metabolism, cognitive performance and depression. J. Am. Med. Dir. Assoc. 2013, 14, 852.e9-852.e17. [CrossRef] [PubMed]

13. Granic, A.; Hill, T.; Kirkwood, T.; Davies, K.; Collerton, J.; Martin-Ruiz, C.; von Zglinicki, T.; Saxby, B.; Wesnes, K.; Collerton, D.; et al. Serum 25-hydroxyvitamin D and cognitive decline in the very old: The Newcastle 85+ Study. Eur. J. Neurol. 2015, 22, 106-115. [CrossRef] 
14. Breitling, L.P.; Perna, L.; Muller, H.; Raum, E.; Kliegel, M.; Brenner, H. Vitamin D and cognitive functioning in the elderly population in Germany. Exp. Gerontol. 2012, 47, 122-127. [CrossRef]

15. Van Schoor, N.; Comijs, H.; Llewellyn, D.; Lips, P. Cross-sectional and longitudinal associations between serum 25-hydroxyvitamin $\mathrm{D}$ and cognitive functioning. Int. Psychogeriatr. 2016, 28, 759-768. [CrossRef]

16. McGrath, J.; Scragg, R.; Chant, D.; Eyles, D.; Burne, T.; Obradovic, D. No association between serum 25-hydroxyvitamin D3 level and performance on psychometric tests in NHANES III. Neuroepidemiology 2007, 29, 49-54. [CrossRef]

17. Maddock, J.; Geoffroy, M.C.; Power, C.; Hypponen, E. 25-Hydroxyvitamin D and cognitive performance in mid-life. Br. J. Nutr. 2014, 111, 904-914. [CrossRef]

18. Lam, V.; Albrecht, M.; Takechi, R.; Prasopsang, P.; Lee, Y.; Foster, J.; Mamo, J. Serum 25-hydroxyvitamin D is associated with reduced verbal episodic memory in healthy, middle-aged and older adults. Eur. J. Nutr. 2016, 55, 1503-1513. [CrossRef]

19. Garcion, E.; Wion-Barbot, N.; Montero-Menei, C.N.; Berger, F.; Wion, D. New clues about vitamin D functions in the nervous system. Trends Endocrinol. Metab. 2002, 13, 100-105. [CrossRef]

20. Annweiler, C.; Schott, A.-M.; Berrut, G.; Chauviré, V.; Le Gall, D.; Inzitari, M.; Beauchet, O. Vitamin D and Ageing: Neurological Issues. Neuropsychobiology. 2010, 62, 139-150. [CrossRef] [PubMed]

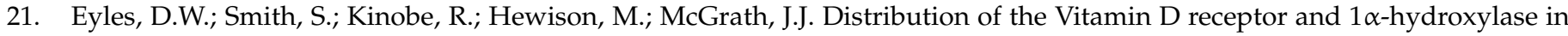
human brain. J. Chem. Neuroanat. 2005, 29, 21-30. [CrossRef] [PubMed]

22. Landel, V.; Annweiler, C.; Millet, P.; Morello, M.; Feron, F. Vitamin D, Cognition and Alzheimer's Disease: The Therapeutic Benefit is in the D-Tails. J. Alzheimer's Dis. 2016, 53, 419-444. [CrossRef] [PubMed]

23. Masoumi, A.; Goldenson, B.; Ghirmai, S.; Avagyan, H.; Zaghi, J.; Abel, K.; Zheng, X.; Espinosa-Jeffrey, A.; Mahanian, M.; Liu, P.T.; et al. 1alpha,25-dihydroxyvitamin D3 interacts with curcuminoids to stimulate amyloid-beta clearance by macrophages of Alzheimer's disease patients. J. Alzheimer's Dis. 2009, 17, 703-717. [CrossRef] [PubMed]

24. Ibi, M.; Sawada, H.; Nakanishi, M.; Kume, T.; Katsuki, H.; Kaneko, S.; Shimohama, S.; Akaike, A. Protective effects of 1 $\alpha, 25-$ $(\mathrm{OH}) 2 \mathrm{D} 3$ against the neurotoxicity of glutamate and reactive oxygen species in mesencephalic culture. Neuropharmacology 2001, 40, 761-771. [CrossRef]

25. Brewer, L.D.; Thibault, V.; Chen, K.-C.; Langub, M.C.; Landfield, P.W.; Porter, N.M. Vitamin D hormone confers neuroprotection in parallel with downregulation of L-type calcium channel expression in hippocampal neurons. J. Neurosci. 2001, 21, 98-108. [CrossRef] [PubMed]

26. Holick, M.F.; Binkley, N.C.; Bischoff-Ferrari, H.A.; Gordon, C.M.; Hanley, D.A.; Heaney, R.P.; Murad, M.H.; Weaver, C.M. Evaluation, Treatment, and Prevention of Vitamin D Deficiency: An Endocrine Society Clinical Practice Guideline. J. Clin. Endocrinol. Metab. 2011, 96, 1911-1930. [CrossRef] [PubMed]

27. Mozos, I.; Marginean, O. Links between Vitamin D deficiency and Cardiovascular Diseases. BioMed Res. Int. 2015, 2015, 109275-109312. [CrossRef]

28. Caraba, A.; Crişan, V.; Romoşan, I.; Mozoş, I.; Murariu, M. Vitamin D status, disease activity, and endothelial dysfunction in early rheumatoid arthritis patients. Dis. Markers. 2017, 2017, 5241012. [CrossRef] [PubMed]

29. Spina, C.S.; Tangpricha, V.; Uskokovic, M.; Adorinic, L.; Maehr, H.; Holick, M.F. Vitamin D and cancer. Anticancer Res. 2006, 26, 2515-2524. [PubMed]

30. Sofianopoulou, E.; Kaptoge, S.K.; Afzal, S.; Jiang, T.; Gill, D.; Gundersen, T.E.; Bolton, T.R.; Allara, E.; Arnold, M.G.; Mason, A.M.; et al. Estimating dose-response relationships for vitamin D with coronary heart disease, stroke, and all-cause mortality: Observational and Mendelian randomisation analyses. Lancet Diabetes Endcrinol. 2021, 9, 837-846. [CrossRef]

31. Zhu, K.; Knuiman, M.; Divitini, M.; Hung, J.; Lim, E.M.; Cooke, B.R.; Walsh, J.P. Serum 25-hydroxyvitamin D as a predictor of mortality and cardiovascular events: A 20-year study of a community-based cohort. Clin. Endocrinol. 2018, 88, 154-163. [CrossRef]

32. Harrell, F.E. Regression Modeling Strategies: With Applications to Linear Models, Logistic Regression, and Survival Analysis; Springer: New York, NY, USA, 2001.

33. James, A.; Hunter, M.; Straker, L.; Beilby, J.; Bucks, R.; Davis, T.; Eikelboom, R.; Hillman, D.; Hui, J.; Hung, J.; et al. Rationale, design and methods for a community-based study of clustering and cumulative effects of chronic disease processes and their effects on ageing: The Busselton healthy ageing study. BMC Public Health. 2013, 13, 936. [CrossRef] [PubMed]

34. Cooke, D.J.; Cooke, B.R.; Bell, D.A.; Vasikaran, S.D.; Glendenning, P. 25-Hydroxyvitamin D C3-epimer is universally present in neonatal Western Australian samples but is unlikely to contribute to diagnostic misclassification. Ann. Clin. Biochem. 2015, 53, 593-598. [CrossRef]

35. Keith, M.S.; Stanislav, S.W.; Wesnes, K.A. Validity of a cognitive computerized assessment system in brain injured patients. Brain Inj. 1998, 12, 1037-1043. [CrossRef]

36. Wesnes, K.A.; Ward, T.; McGinty, A.; Petrini, O. The memory enhancing effects of a Ginkgo biloba/Panax ginseng combination in healthy middle-aged volunteers. Psychopharmacology 2000, 152, 353-361. [CrossRef]

37. Folstein, M.F.; Folstein, S.E.; McHugh, P.R. "Mini-mental state": A practical method for grading the cognitive state of patients for the clinician. J. Psychiatr. Res. 1975, 12, 189-198. [CrossRef]

38. Nelson, H.E.; Willison, J. National Adult Reading Test Manual, 2nd ed.; NFER-Nelson: Windsor, UK, 1991.

39. Booth, M. Assessment of Physical Activity: An International Perspective. Res. Q. Exerc. Sport. 2015, 71, 114-120. [CrossRef] [PubMed] 
40. Kroenke, K.; Spitzer, R.L.; Williams, J.B. The PHQ-9: Validity of a brief depression severity measure. J. Gen. Intern. Med. 2001, 16, 606-613. [CrossRef] [PubMed]

41. Spitzer, R.L.; Kroenke, K.; Williams, J.B.W.; Löwe, B. A Brief Measure for Assessing Generalized Anxiety Disorder: The GAD-7. Arch. Intern. Med. 2006, 166, 1092-1097. [CrossRef]

42. Lovibond, S.H.; Lovibond, P.F. Manual for the Depression Anxiety Stress Scales, 2nd ed.; Psychology Foundation: Sydney, Australia, 1995.

43. Shoben, A.B.; Kestenbaum, B.; Levin, G.; Hoofnagle, A.N.; Psaty, B.M.; Siscovick, D.S.; de Boer, I.H. Seasonal Variation in 25-Hydroxyvitamin D Concentrations in the Cardiovascular Health Study. Am. J. Epidemiol. 2011, 174, 1363-1372. [CrossRef] [PubMed]

44. Sachs, M.C.; Shoben, A.; Levin, G.P.; Robinson-Cohen, C.; Hoofnagle, A.N.; Swords-Jenny, N.; Ix, J.H.; Budoff, M.; Lutsey, P.L.; Siscovick, D.S.; et al. Estimating mean annual 25-hydroxyvitamin D concentrations from single measurements: The Multi-Ethnic Study of Atherosclerosis. Am. J. Clin. Nutr. 2013, 97, 1243-1251. [CrossRef] [PubMed]

45. Watt, S.; Ong, B.; Crowe, S.F. Developing a regression equation for predicting premorbid functioning in an Australian sample using the National Adult Reading Test: Predicting premorbid functioning. Aust. J. Psychol. 2018, 70, 186-195. [CrossRef]

46. Lerchbaum, E. Vitamin D and menopause-A narrative review. Maturitas 2014, 79, 3-7. [CrossRef] [PubMed]

47. Peterson, A.; Mattek, N.; Clemons, A.; Bowman, G.L.; Buracchio, T.; Kaye, J.; Quinn, J. Serum vitamin D concentrations are associated with falling and cognitive function in older adults. J. Nutr. Health Aging 2012, 16, 898-901. [CrossRef]

48. Feart, C.; Helmer, C.; Merle, B.; Herrmann, F.R.; Annweiler, C.; Dartigues, J.F.; Delcourt, C.; Samieri, C. Associations of lower vitamin D concentrations with cognitive decline and long-term risk of dementia and Alzheimer's disease in older adults. Alzheimers. Dememt. 2017, 13, 1207-1216. [CrossRef]

49. Llewellyn, D.J.; Langa, K.M.; Lang, I.A. Serum 25-hydroxyvitamin D concentration and cognitive impairment. J. Geriatr. Psychiatry Neurol. 2009, 22, 188-195. [CrossRef] [PubMed]

50. Jorde, R.; Mathiesen, E.B.; Rogne, S.; Wilsgaard, T.; Kjaergaard, M.; Grimnes, G.; Schirmer, H. Vitamin D and cognitive function: The Tromso Study. J. Neurol. Sci. 2015, 355, 155-161. [CrossRef]

51. Miller, J.; Harvey, D.; Beckett, L.A.; Green, R.; Farias, S.T.; Reed, B.; Olichney, J.; Mungas, D.; DeCarli, C. Vitamin D status predicts rates of cognitive decline in a multi-ethnic cohort of older adults. JAMA Neurol. 2015, 72, 1295-1303. [CrossRef] [PubMed]

52. Cui, X.; Gooch, H.; Groves, N.J.; Sah, P.; Burne, T.H.; Eyles, D.W.; McGrath, J.J. Vitamin D and the brain: Key questions for future research. J. Steriod Biochem. Mol. 2015, 148, 305-309. [CrossRef] [PubMed]

53. Nabe-Nielsen, K.; Holtermann, A.; Gyntelberg, F.; Garde, A.H.; Islamoska, S.; Prescott, E.; Schnohr, P.; Hansen, Å.M. The effect of occupational physical activity on dementia: Results from the Copenhagen Male Study. Scand. J. Med. Sci. Sports 2021, 31, 446-455. [CrossRef] [PubMed] 